\title{
Comparison between Two Radiological Methods for Assessment of Tooth Root Resorption: An In Vitro Study
}

\author{
Sabina Saccomanno, Pier Carmine Passarelli $\mathbb{D}^{D}$, Bruno Oliva, and Cristina Grippaudo \\ Department of Dental Clinic, Catholic University of Sacred Heart of Rome, Rome, Italy \\ Correspondence should be addressed to Pier Carmine Passarelli; piercarminepassarelli@hotmail.it
}

Received 9 December 2017; Revised 24 January 2018; Accepted 5 February 2018; Published 4 March 2018

Academic Editor: Roberto Cameriere

Copyright ( 2018 Sabina Saccomanno et al. This is an open access article distributed under the Creative Commons Attribution License, which permits unrestricted use, distribution, and reproduction in any medium, provided the original work is properly cited.

Purpose. This study aims to verify the validity of the radiographic image and the most effective radiological techniques for the diagnosis of root resorption to prevent, cure, and reduce it and to verify if radiological images can be helpful in medical and legal situations. Methods. 19 dental elements without root resorption extracted from several patients were examined: endooral and panoramic radiographs were performed, with traditional and digital methods. Then the root of each tooth was dipped into 3-4 mm of $10 \%$ nitric acid for 24 hours to simulate the resorption of the root and later submitted again to radiological examinations and measurements using the same criteria and methods. Results. For teeth with root resorption the real measurements and the values obtained with endooral techniques and digital sensors are almost the same, while image values obtained by panoramic radiographs are more distorted than the real ones. Conclusions. Panoramic radiographs are not useful for the diagnosis of root resorption. The endooral examination is, in medical and legal fields, the most valid and objective instrument to detect root resorption. Although the literature suggests that $\mathrm{CBCT}$ is a reliable tool in detecting root resorption defects, the increased radiation dosage and expense and the limited availability of $\mathrm{CBCT}$ in most clinical settings accentuate the outcome of this study.

\section{Introduction}

Histologically root resorption is an irreversible demineralization of the cementum (sometimes of the dentin) of the surface of the root of a tooth [1]. Diagnosis can be done by anamnestic data and careful clinical observation, but only radiological examinations are determinant, often exclusive, and usually conclusive. Causes of root resorption can be general or local: endocrine pathologies, significant oral dysfunctions, osteoporosis, traumas or external causes (orthodontic treatments), expanders, intrusion movements, aggressive or inappropriate orthodontic therapy, and therapy in patients with predisposition for root resorption (traumas, osteoporosis, or hypothyroidism) [2-4].

There are several theories about root resorption: Becks et al. $[5,6]$ wrote about hereditary transmission of the individual predisposition for root resorption. Rygh et al. [7] write about predisposition not only in different individuals, but also in the same person at different times, as hormone metabolical signals can change the osteoblastic/osteoclastic activity.
The age of the patient is important: the greatest orthodontic resorption is noticeable during adolescence when more orthodontic treatments are made and the apexes are already closed. Sameshima and Sinclair [8] stress that radicular resorption increases with age, especially in the anterior segment, corresponding to the incisors. Melsen et al. [9] assert that adults do not have the same cellular pool of young people and that is why forces applied to teeth through orthodontic treatment should be reduced, because the quantity of bone that must be resorbed in relation to a peculiar dental movement is also reduced.

Most authors [10] find no connection between sex and root resorption, although Brezniak and Wasserstein [11, 12] assert that females are more incline to idiopathic radicular reabsorption to the ratio of $3.7: 1$.

Specific radiological examinations can be valid documentation especially for orthodontists who, more than other specialists, are often subject to medical-legal jurisdiction concerning a contentious resorption of root. The radiologic examinations are actually an impartial permanent document 
that in the medical-legal field can be evaluated by several examiners, more independently than other subjective clinical evaluations [13, 14]. Radiologic examinations can reveal a pathology before the clinical symptoms appear, which is important, especially if an inappropriate orthodontic treatments can be avoided, if it could worsen the conditions of the teeth and their roots.

Teeth, because of their high density, are extremely radiopaque and radiographically well defined; therefore, endooral radiography (periapical radiographs) and extraoral radiography (orthopanoramic or OPG), based on both traditional and digital methods, can give useful information to the specialist $[2,15,16]$. Although the Cone Beam Computerized Tomography (CBCT) has become a very important tool in diagnostics, our study focused primarily on 2D digital analysis. Therefore we did not include CBCT analysis of root resorption in this study.

\subsection{Traditional Method}

1.1.1. Orthopanoramic Technique (OPG). It visualizes wide surfaces $13 \times 18 / 18 \times 24 / 24 \times 30 \mathrm{~cm}$ and it is made by an orthopantomograph in which the X-ray origin and the film box rotate simultaneously while the patient remains still with his/her chin on the appropriate support [17]. It is useful as a first diagnostic orientation and offers a general image of teeth, arches, maxillary bones, paranasal sinuses and TMJ but, because of its approximate definition and dental overlap, it does not provide the same anatomical details obtained with endooral radiography.

1.1.2. Endooral Technique. It visualizes smaller surfaces $2 \times$ $3 / 3 \times 4 / 4 \times 5 / 5 \times 7 \mathrm{~cm}$ and offers more accurate radiographic data and details about the dental alveoli. The exact image of the examined structure is not obtained, because the image is deformed by the angulations of rays that come from a single point but conic origin.

To reduce deformations, the film must be positioned parallel and near the structure to impress, so that the central ray of the beam can hit perpendicularly both the structure and the film.

1.2. Digital Method. This method is used for both the endooral (endooral RX) and extraoral (OPG) techniques.

Digital radiology assigns a numerical value to the different levels of X-ray absorption in the tissues and to differentiate the several tones of grey that compose the radiographic image. Digital radiology is divided into inline digital radiology, if the numerical image can be obtained directly from the patient, and into outline digital radiology, if the numerical image is obtained by a previously made diagram. The reduction in radiation dosage in digital radiology, the quality of images, and the postprocessing elaboration and optimization are all parts of an important progress in dental radiology.

This study aims to verify the validity of the radiographic image and the most effective radiological techniques for the diagnosis of rizalysis to prevent, cure, and reduce damage or reabsorption of the root. This study aims also to verify

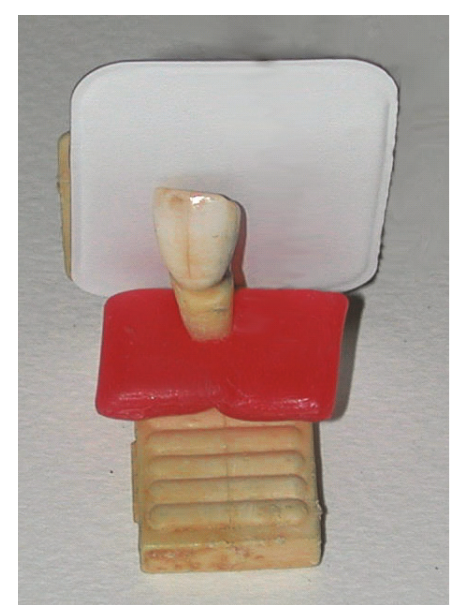

FiguRE 1: Extracted tooth on the endooral centering support.

if radiological images can be helpful in medical and legal situations.

\section{Materials and Methods}

We examined a sample of 19 dental elements without root resorption extracted from several patients and for different reasons; each element has been measured in decimals of millimeter by caliper.

We used a long cone radiographic apparatus $(70 \mathrm{kv}$ and $7 \mathrm{~mA}$ ) with a constant tension and constant times of exposure $(0.12 \mathrm{sec}$ for the traditional methods and $0.10 \mathrm{sec}$ for digital sensors).

We used a standardizing centering support (Figure 1) on which teeth have been positioned and fixed by orthodontic wax; then we placed the centering support $20 \mathrm{~cm}$ away from the X-ray origin and took radiographs of each tooth using both methods. The apex of the root and coronal border of each tooth were our reference marks and the measurements with a caliper were made after drawing the parallel tangent lines to these points.

We later created a simulated dental arch made of orthodontic resin, methacrylic orthocryl (Figure 2), and to recreate a thickness simulating soft tissues we used a common medical gauze. Then orthopanoramic radiographic examinations (OPG), a traditional method, were applied.

The examinations were made with a $60 \mathrm{Kv}$ and $9.0 \mathrm{~mA} / 15 \mathrm{sec}$ orthopantomograph, because of the material used for the simulated dental arch. It was not possible to lower these parameters and so we decided to superimpose three films in the box to obtain three images and to have the possibility of choosing the best one. Digital measurements were made using a calibrated system from an image derived from a computerized program (Image J).

Then the root of each tooth was dipped into 3-4 $\mathrm{mm}$ of $10 \%$ nitric acid for 24 hours to simulate of resorption of the root and later submitted again to radiological examinations and measurements using the same criteria and methods (Figures 3, 4, and 5). The measurements obtained were then statistically compared. 


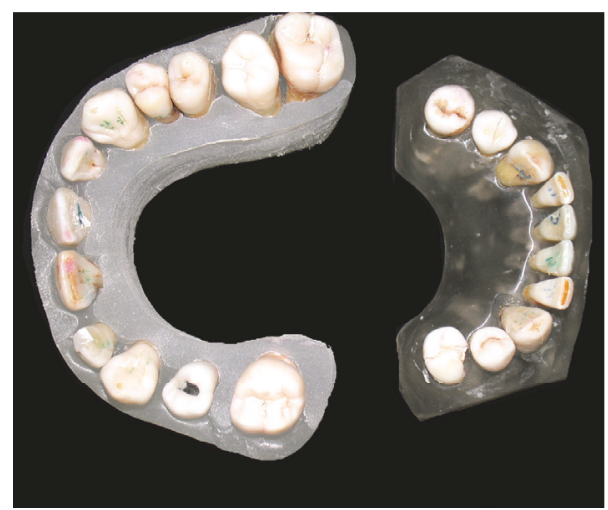

FIGURE 2: Simulated dental arch made of orthodontic resin, methacrylic orthocryl: occlusal vision.

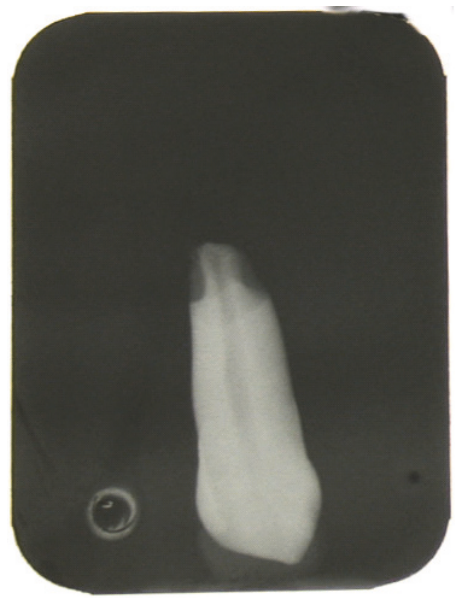

FIGURE 3: Traditional endooral X-ray: rizalysis.

All the data collected was processed by a statistical program to calculate the percentage of the standard deviation of the values obtained with different radiological methods, before and after reabsorption of the root.

\section{Results}

Using the obtained data, recorded in Tables 1 and 2, we draw some curves whose observation led us to the following results:

(i) The most reliable radiological method is the endooral technique because the values we obtained are more homogeneous and more similar to the real ones.

(ii) The extraoral technique (OPG) is the least reliable, as it gives a bigger image that distorts the original size. Plus, it does not offer precise anatomic details because of the presence of reinforcement protections that lowers the quantity of radiations needed to impress the image. The resulting tables show how the percentage of the deformation values has increased when compared to the endooral projections.

(iii) If we compare both tables, we notice that the values of the teeth submitted to rizalysis and then analyzed

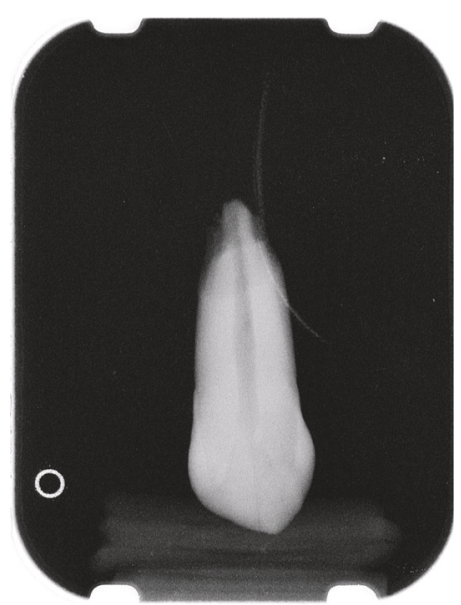

FIGURE 4: Digital endooral X-ray: rizalysis.

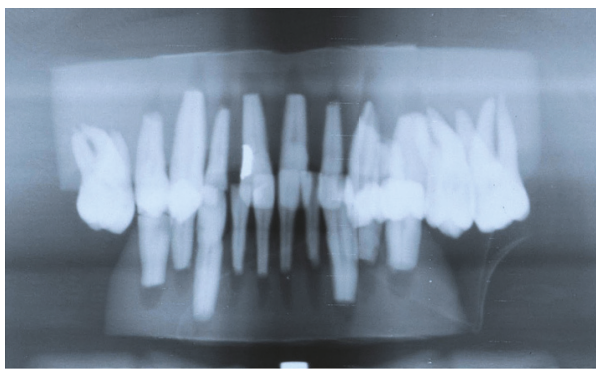

FIGURE 5: Panoramic radiograph: rizalysis.

with extraoral method (OPG) have increased image deformation.

(iv) For teeth with root resorption the real values and the values obtained with endooral technique and digital sensors are almost the same, while image values obtained by extraoral methods are more distorted than the real ones.

\section{Discussion}

This study for the diagnosis of the radicular resorption has been made comparing various radiographic methods and techniques in vitro and shows the advantages and disadvantages of each method and technique.

The endooral technique provides a better qualitative and quantitative analysis than the orthopanoramic one. The limits are the absence of a standard process and the distortion of the endooral projections using the bisecting method: if the ray beam has a juxta-gingival or parallel orientation, the dental roots appear longer, while if the beam exceeds the apexes, the roots appear shorter.

The examinations by OPG provide less than neat images, with a bigger distorting enlargement and that is why OPG is not useful for the diagnosis of root resorption, but rather it is used to exclude other dental causes (agenesis, inclusion, or ectopy). It cannot be considered a valid diagnostic and medical-legal examination because at the level of the anterior 
TABLE 1: Tooth length without tooth resorption.

\begin{tabular}{|c|c|c|c|c|c|c|c|}
\hline \multirow[t]{2}{*}{ Tooth number } & \multirow{2}{*}{$\begin{array}{c}\text { Real tooth } \\
\text { length } \\
\text { Total }\end{array}$} & \multicolumn{2}{|c|}{$\begin{array}{l}\text { Radiological measurements } \\
\text { using traditional endooral X-rays }\end{array}$} & \multicolumn{2}{|c|}{$\begin{array}{l}\text { Radiological measurements } \\
\text { using panoramic radiographs }\end{array}$} & \multicolumn{2}{|c|}{$\begin{array}{l}\text { Radiological measurements } \\
\text { using digital endooral X-rays }\end{array}$} \\
\hline & & Var.\% & Total & Var.\% & Total & Var.\% & Total \\
\hline 11 & 24.84 & $4.31 \%$ & 25.91 & $8.86 \%$ & 27.04 & $3.38 \%$ & 25.68 \\
\hline 12 & 21.80 & $5.73 \%$ & 23.05 & $11.56 \%$ & 24.32 & $5.47 \%$ & 22.99 \\
\hline 13 & 28.06 & $4.49 \%$ & 29.32 & $10.83 \%$ & 31.10 & $5.63 \%$ & 29.64 \\
\hline 14 & 24.60 & $1.38 \%$ & 24.94 & $9.02 \%$ & 26.82 & $0.41 \%$ & 24.70 \\
\hline 21 & 24.86 & $4.42 \%$ & 25.96 & $13.19 \%$ & 28.14 & $2.96 \%$ & 25.60 \\
\hline 22 & 22.50 & $3.60 \%$ & 23.31 & $12.53 \%$ & 25.32 & $12.94 \%$ & 25.41 \\
\hline 23 & 28.24 & $4.92 \%$ & 29.63 & $11.19 \%$ & 31.40 & $4.25 \%$ & 29.44 \\
\hline 24 & 20.20 & $7.13 \%$ & 21.64 & $5.45 \%$ & 21.30 & $5.64 \%$ & 21.34 \\
\hline 25 & 22.60 & $3.54 \%$ & 23.40 & $11.50 \%$ & 25.20 & $5.78 \%$ & 23.91 \\
\hline 31 & 21.80 & $3.67 \%$ & 22.60 & $13.30 \%$ & 24.70 & $3.04 \%$ & 22.46 \\
\hline 32 & 22.16 & $4.74 \%$ & 23.21 & $18.41 \%$ & 26.24 & $1.89 \%$ & 22.58 \\
\hline 33 & 26.82 & $2.68 \%$ & 27.54 & $23.79 \%$ & 33.20 & $0.74 \%$ & 27.02 \\
\hline 34 & 19.74 & $6.69 \%$ & 21.06 & $21.58 \%$ & 24.00 & $1.89 \%$ & 20.11 \\
\hline 35 & 20.14 & $2.78 \%$ & 20.70 & $11.22 \%$ & 22.40 & $1.54 \%$ & 20.45 \\
\hline 41 & 21.30 & $9.86 \%$ & 23.40 & $16.81 \%$ & 24.88 & $5.32 \%$ & 22.43 \\
\hline 42 & 22.38 & $3.22 \%$ & 23.10 & $13.14 \%$ & 25.32 & $0.18 \%$ & 22.42 \\
\hline 43 & 27.70 & $4.12 \%$ & 28.84 & $15.81 \%$ & 32.08 & $0.36 \%$ & 27.80 \\
\hline 44 & 20.76 & $4.91 \%$ & 21.78 & $13.68 \%$ & 23.60 & $0.29 \%$ & 20.82 \\
\hline 45 & 20.20 & $4.46 \%$ & 21.10 & $20.89 \%$ & 24.42 & $5.63 \%$ & 21.34 \\
\hline
\end{tabular}

TABLE 2: Tooth length with rizalysis.

\begin{tabular}{|c|c|c|c|c|c|c|c|}
\hline \multirow[t]{2}{*}{ Tooth number } & \multirow{2}{*}{$\begin{array}{c}\text { Real tooth } \\
\text { length } \\
\text { Total }\end{array}$} & \multicolumn{2}{|c|}{$\begin{array}{l}\text { Radiological measurements } \\
\text { using traditional endooral X-rays }\end{array}$} & \multicolumn{2}{|c|}{$\begin{array}{l}\text { Radiological measurements } \\
\text { using panoramic radiographs }\end{array}$} & \multicolumn{2}{|c|}{$\begin{array}{l}\text { Radiological measurements } \\
\text { using digital endooral X-rays }\end{array}$} \\
\hline & & Var.\% & Total & Var.\% & Total & Var.\% & Total \\
\hline 11 & 19.72 & $2.03 \%$ & 20.12 & $12.07 \%$ & 22.10 & $1.70 \%$ & 20.06 \\
\hline 12 & 18.72 & $3.74 \%$ & 19.42 & $18.59 \%$ & 22.20 & $0.56 \%$ & 18.82 \\
\hline 13 & 23.88 & $0.42 \%$ & 23.98 & $13.15 \%$ & 27.02 & $0.06 \%$ & 23.90 \\
\hline 14 & 15.25 & $13.18 \%$ & 17.26 & $32.59 \%$ & 20.22 & $1.39 \%$ & 15.46 \\
\hline 21 & 19.62 & $2.55 \%$ & 20.12 & $18.25 \%$ & 23.20 & $2.91 \%$ & 20.19 \\
\hline 22 & 17.38 & $1.73 \%$ & 17.68 & $12.08 \%$ & 19.48 & $1.06 \%$ & 17.56 \\
\hline 23 & 22.24 & $2.11 \%$ & 22.71 & $8.27 \%$ & 24.08 & $32.38 \%$ & 29.44 \\
\hline 24 & 15.25 & $4.26 \%$ & 15.90 & $23.80 \%$ & 18.88 & $1.39 \%$ & 15.46 \\
\hline 25 & 18.22 & $1.43 \%$ & 18.48 & $21.41 \%$ & 22.12 & $1.02 \%$ & 18.41 \\
\hline 31 & 16.72 & $0.60 \%$ & 16.82 & $22.61 \%$ & 20.50 & $0.42 \%$ & 16.79 \\
\hline 32 & 17.62 & $0.57 \%$ & 17.72 & $16.40 \%$ & 20.51 & $0.46 \%$ & 17.70 \\
\hline 33 & 21.40 & $0.93 \%$ & 21.60 & $20.19 \%$ & 25.72 & $0.47 \%$ & 21.50 \\
\hline 34 & 13.36 & $4.79 \%$ & 14.00 & $16.62 \%$ & 15.58 & $0.49 \%$ & 13.43 \\
\hline 35 & 15.21 & $1.78 \%$ & 15.48 & $17.69 \%$ & 17.90 & $0.36 \%$ & 15.27 \\
\hline 41 & 17.54 & $2.05 \%$ & 17.90 & $25.43 \%$ & 22.00 & $0.57 \%$ & 17.64 \\
\hline 42 & 15.70 & $2.55 \%$ & 16.10 & $16.94 \%$ & 18.36 & $0.36 \%$ & 15.76 \\
\hline 43 & 23.29 & $0.64 \%$ & 23.44 & $14.21 \%$ & 26.60 & $0.36 \%$ & 23.37 \\
\hline 44 & 15.50 & $2.84 \%$ & 15.94 & $18.45 \%$ & 18.36 & $0.40 \%$ & 15.56 \\
\hline 45 & 16.20 & $6.79 \%$ & 17.30 & $21.11 \%$ & 19.62 & $1.58 \%$ & 16.46 \\
\hline
\end{tabular}


part of the maxilla and the mandible, corresponding with the incisor teeth, there is a thinning of the tomographic section, exactly where the thickness should be as great as possible. If we combine all these shortcomings, the OPG exam becomes totally useless to assess root resorption. Therefore, studying root resorption using OPG, at the end of an orthodontic treatment, for instance, is not a very accurate method because of the distortion of the image and the variation of the dental inclination, which is also evident in the endooral projections $[18,19]$.

It is of paramount importance to have a wide thickness of the section examined, for the correct and full vision of the anterior elements on the OPG radiogram. This section must be as wide as possible to reproduce the total vertical dimension of maxillary and mandibular incisors because in the anterior region there is a thinning of the tomographic section that, in people with an increased interincisor angle, is more significant than the average, so there is a need for a particular image of these elements [20].

Moreover, if the OPG is executed with a digital method it is possible to have greater diagnostic precision for specific anatomic structures, whose interfaces are better shown by the border effect: superior and inferior frontal teeth, radicular apexes, nasal choanae floor of the maxillary sinuses, nasal and maxillary sinus septum, nasal septum, and mandibular condyles. The digital OPG examinations produce less distorted images and shades, so typical in the topographic method, with the dosage saving being also a positive aspect.

In this study we obtained very good images of the coronal and radicular structures by periapical X-rays and identified well the simulated radicular lesion. In addition, the compared values of the measurements are more similar to the real ones. For these reasons we think that the orthodontist should also prescribe, along with normal routine examinations, periapical endooral RX of incisors, canines, and premolars (these teeth are most subject to root resorption) with the $\mathrm{X}$ ray tube positioner in place for the orthogonality of the rays in relation to the film and for the constant preservation of the right distance between teeth and X-ray origin [21].

Digital radiography gives us more definite images of crowns and roots using the wide grey scale and the possibility of elaborating the obtained data in postprocessing: the dose of radiation is low, the images are more reliable and immediately visualizable, and the operator can modify them to show details, enlarge them, measure, and easily file them to be used when necessary.

The conventional radiographic methods give bidimensional images and do not allow a proper vision of the lacunae of minor reabsorption. Since the Cone Beam Computerized Tomography (CBCT) might be useful for enhancing diagnosis of early root resorption, there is a need to compare the diagnostic accuracy of the CBCT images with digital periapical radiographs for assessing root defects. The CBCT is effective and reliable in detecting the presence of resorption lesions, although digital intraoral radiography results in an acceptable level of accuracy [22].

Although the literature suggests that CBCT is a reliable tool in detecting root resorption defects, it is rare that studies compare CBCT efficacy with intraoral digital 2D modalities.
Kumar et al. [23] showed that examiners performed slightly weaker in the detection of root defects when using CBCT images compared with conventional periapical radiographs. The increased radiation dosage and expense and the limited availability of CBCT in most clinical settings accentuate the outcome of this study, which is to confirm that periapical radiographs are better at detecting root defects. However, when a full-mouth series or periapical radiographs are not available and a CBCT scan of the patient is already available, the CBCT data could be used to detect root resorption via $2 \mathrm{D}$ or $3 \mathrm{D}$ evaluation without additional radiation exposure. The lack of superiority of either imaging modality suggests that periapical or CBCT imaging can be used to identify root defects. However, because of the increased radiation exposure from CBCT, using CBCT for identifying defects should be considered with the caveat that CBCT data is already available for analysis [23]. Sousa Melo et al. [24] simulated "early stage" external root resorption lesions in the apical third of anterior teeth: they suggested there was a considerable difference between tomographic images with the most common voxel size used in orthodontics $(0.4 \mathrm{~mm})$ and those with a smaller voxel size $(0.125 \mathrm{~mm})$, suggesting that a more dedicated, high-resolution scan should be acquired when one intends to investigate the early stage of external root resorption during orthodontic treatment. This method could be the most reliable approach in detecting root resorption but the selection of voxel size increases the radiation exposure [25, 26]. Lima et al. [27] studied root resorption after dental trauma comparing periapical radiography and CBCT: they confirmed the superiority of CBCT in identifying it. However, they also reported that CBCT should not be used routinely for diagnosing root resorption, but it is recommended when lesion is suspected and more information about the shape of the defects is needed. All exposure to ionizing radiation should follow the "as low as reasonably achievable" principle and for this reason, the selection criteria and the parameters for each CBCT scan protocol should be strict and follow the respective clinical indication. Some limitations are associated with this study: diagnostic test may be affected by observer performance and experience, hardware and software specification, and viewing conditions; in in vitro study there are no nuances of human soft tissue attenuation artifacts. One limitation of our study was the small sample size. The size was based on previous root resorption research [22, 23]. Further studies with a bigger sample size than ours are requested to confirm these results.

\section{Conclusions}

Radiologic examinations can notice the pathology before the clinical symptoms appear, especially useful if we can avoid inappropriate orthodontic treatments which could worsen the conditions of the teeth and their roots.

All teeth submitted for orthodontic treatment may have a very small amount of resorption that is clinically insignificant and radiologically invisible. This kind of resorption usually does not influence the functional activity and life of the tooth because it stops as soon as the active treatment is finished. This range of resorption of root can be considered a small 
price to pay if compared with all the advantages of a well-done orthodontic treatment.

From our study, it may be inferred that the endooral examination is, in medical and legal fields, the most valid and objective instrument to detect root resorption. Although a more advanced method such as the CBCT may yield similar results, the device is not suitable for most working realities or even for most clinics worldwide, confirming the traditional endooral X-ray as the best option to examine root resorption in a wide variety of situations, especially in case of elective orthodontic treatment.

\section{Conflicts of Interest}

The authors declare that there are no conflicts of interest regarding the publication of this paper.

\section{References}

[1] S. E. Bishara, L. Vonwald, and J. R. Jakobsen, "Changes in root length from early to mid-adulthood: resorption or apposition?" American journal of orthodontics and dentofacial orthopedics : official publication of the American Association of Orthodontists, its constituent societies, and the American Board of Orthodontics, vol. 115, no. 5, pp. 563-568, 1999.

[2] S. A. Alexander, "Levels of root resorption associated with continuous arch and sectional arch mechanics.", American journal of orthodontics and dentofacial orthopedics : official publication of the American Association of Orthodontists, its constituent societies, and the American Board of Orthodontics, vol. 110, no. 3, pp. 321-324, 1996.

[3] S. Alwali, M. Marklund, and M. Persson, "Apical root resorption of upper first molars as related to anchorage system," Swedish Dental Journal, vol. 24, no. 4, pp. 145-153, 2000.

[4] S. Baumrind, E. L. Korn, and R. L. Boyd, "Apical root resorption in orthodontically treated adults.", American journal of orthodontics and dentofacial orthopedics : official publication of the American Association of Orthodontists, its constituent societies, and the American Board of Orthodontics, vol. 110, no. 3, pp. 311-320, 1996.

[5] H. Becks and J. A. Marshall, "Resorption or Absorption?" The Journal of the American Dental Association, vol. 19, pp. 15281537, 1932.

[6] H. Becks and R. C. Cowden, "Root resorptions and their relation to pathologic bone formation. Part II. Classification, degrees, prognosis and frequency," American Journal of Orthodontics and Dentofacial Orthopedics, vol. 28, no. 8, pp. 513-526, 1942.

[7] P. Rygh, K. Bowling, L. Hovlandsdal, and S. Williams, "Activation of the vascular system: A main mediator of periodontal fiber remodeling in orthodontic tooth movement," American Journal of Orthodontics and Dentofacial Orthopedics, vol. 89, no. 6, pp. 453-468, 1986.

[8] G. T. Sameshima and P. M. Sinclair, "Predicting and preventing root resorption: part I. Diagnostic factors," American Journal of Orthodontics and Dentofacial Orthopedics, vol. 119, no. 5, pp. 505-510, 2001.

[9] B. Melsen, N. Agerbæk, J. Erikson, and S. Terp, "New attachment through periodontal treatment and orthodontic intrusion," American Journal of Orthodontics and Dentofacial Orthopedics, vol. 94, no. 2, pp. 104-116, 1988.
[10] P. Brudvik and P. Rygh, "Transition and determinants of orthodontic root resorption-repair sequence," European Journal of Orthodontics, vol. 17, no. 3, pp. 177-188, 1995.

[11] N. Brezniak and A. Wasserstein, "Root resorption after orthodontic treatment: Part 1. Literature review," American Journal of Orthodontics and Dentofacial Orthopedics, vol. 103, no. 1, pp. 62-66, 1993.

[12] N. Brezniak and A. Wasserstein, "Root resorption after orthodontic treatment: part 2. Literature review," American Journal of Orthodontics and Dentofacial Orthopedics, vol. 103, no. 2, pp. 138-146, 1993.

[13] M. Rivera, S. De Luca, L. Aguilar, L. A. Velandia Palacio, I. Galić, and R. Cameriere, "Measurement of open apices in tooth roots in Colombian children as a tool for human identification in asylum and criminal proceedings," Journal of Forensic and Legal Medicine, vol. 48, pp. 9-14, 2017.

[14] R. Cameriere, A. Pacifici, L. Pacifici et al., "Age estimation in children by measurement of open apices in teeth with Bayesian calibration approach," Forensic Science International, vol. 258, pp. 50-54, 2016.

[15] B. M. Thomson, J. Saklatvala, and T. J. Chambers, "Osteoblasts mediate interleukin 1 stimulation of bone resorption by rat osteoclasts," The Journal of Experimental Medicine, vol. 164, no. 1, pp. 104-112, 1986.

[16] L. Tronstad, "Root resorption, A multidisciplinary problem in dentistry. In Davidovitch Z: Biological mechanism of tooth eruption and root resorption," Ebsco Media, Birmingham Ala, pp. 293-302, 1988.

[17] K. L. Andersen, E. H. Pedersen, and B. Melsen, "Material parameters and stress profiles within the periodontal ligament," American Journal of Orthodontics and Dentofacial Orthopedics, vol. 99, no. 5, pp. 427-440, 1991.

[18] T. S. Taylor, R. J. Ackerman Jr., and P. K. Hardman, "Exposure reduction and image quality in orthodontic radiology: A review of the literature," American Journal of Orthodontics and Dentofacial Orthopedics, vol. 93, no. 1, pp. 68-77, 1988.

[19] T. Lauc, E. Nakaš, M. Latić-Dautović et al., "Dental Age in Orthodontic Patients with Different Skeletal Patterns," BioMed Research International, vol. 2017, Article ID 8976284, 2017.

[20] F. M. Andreasen, I. Sewerin, U. Mandel, and J. O. Andreasen, "Radiographic assessment of simulated root resorption cavities," Dental Traumatology, vol. 3, no. 1, pp. 21-27, 1987.

[21] L. Feller, R. A. G. Khammissa, G. Thomadakis, J. Fourie, and J. Lemmer, "Apical External Root Resorption and Repair in Orthodontic Tooth Movement: Biological Events," BioMed Research International, vol. 2016, Article ID 4864195, 2016.

[22] S. Patel, A. Dawood, R. Wilson, K. Horner, and F. Mannocci, "The detection and management of root resorption lesions using intraoral radiography and cone beam computed tomography-an in vivo investigation," International Endodontic Journal, vol. 42, no. 9, pp. 831-838, 2009.

[23] V. Kumar, L. Gossett, A. Blattner, L. R. Iwasaki, K. Williams, and J. C. Nickel, "Comparison between cone-beam computed tomography and intraoral digital radiography for assessment of tooth root lesions," American Journal of Orthodontics and Dentofacial Orthopedics, vol. 139, no. 6, pp. e533-e541, 2011.

[24] S. L. Sousa Melo, K. D. F. Vasconcelos, N. Holton et al., "Impact of cone-beam computed tomography scan mode on the diagnostic yield of chemically simulated external root resorption," American Journal of Orthodontics and Dentofacial Orthopedics, vol. 151, no. 6, pp. 1073-1082, 2017. 
[25] D. Brüllmann and R. K. W. Schulze, "Spatial resolution in CBCT machines for dental/maxillofacial applications - What do we know today?" Dentomaxillofacial Radiology, vol. 44, no. 1, Article ID 20140204, 2015.

[26] R. Pauwels, K. Araki, J. H. Siewerdsen, and S. S. Thongvigitmanee, "Technical aspects of dental CBCT: State of the art," Dentomaxillofacial Radiology, vol. 44, no. 1, Article ID $20140224,2015$.

[27] T. F. Lima, T. O. Gamba, A. A. Zaia, and A. J. Soares, "Evaluation of cone beam computed tomography and periapical radiography in the diagnosis of root resorption," Australian Dental Journal, vol. 61, no. 4, pp. 425-431, 2016. 


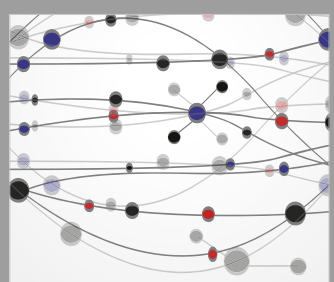

The Scientific World Journal
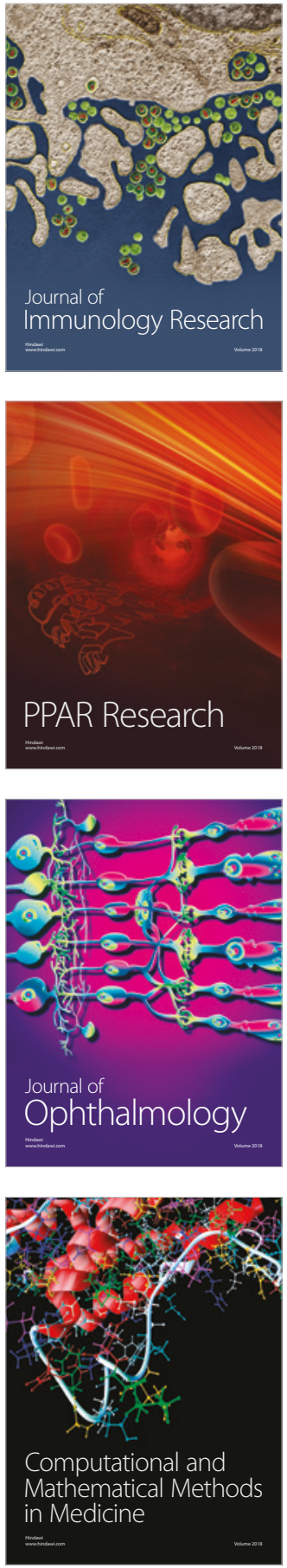

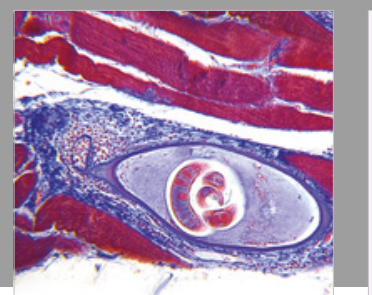

Gastroenterology Research and Practice

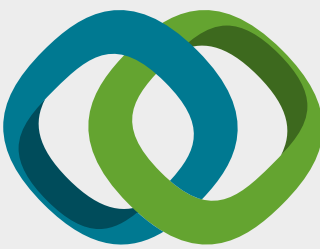

\section{Hindawi}

Submit your manuscripts at

www.hindawi.com
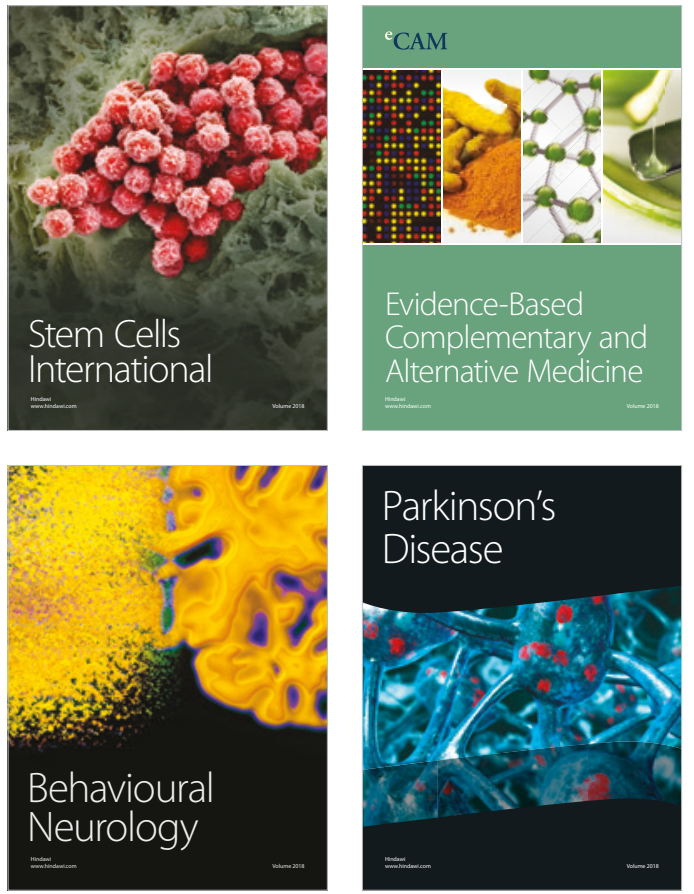

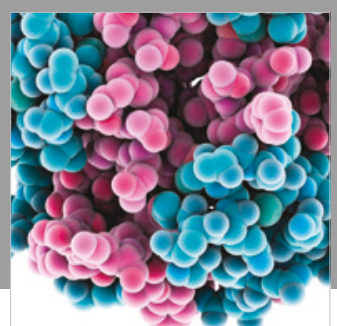

ournal of

Diabetes Research

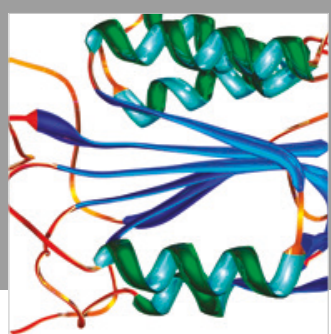

Disease Markers
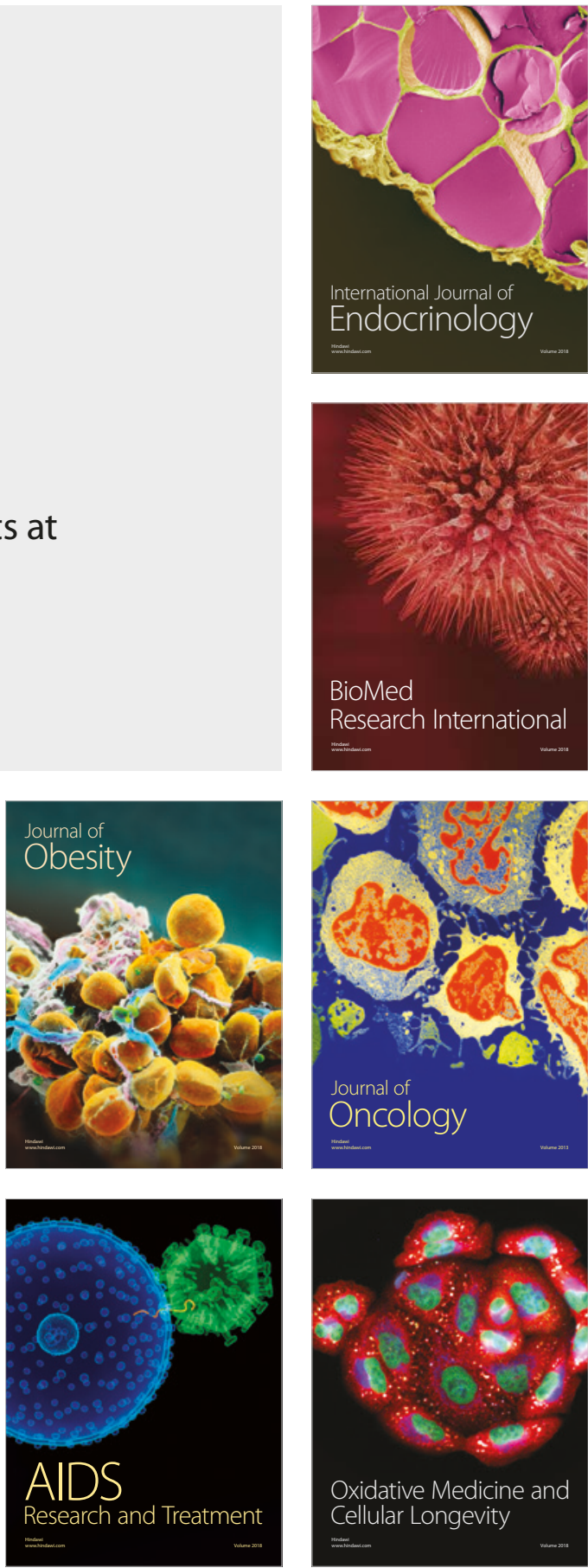